\title{
Secular Trends in the Clinical Characteristics of Type 2 Diabetic Patients With Severe Hypoglycemia Between 2008 and 2013
}

\author{
Hiroyuki Ito ${ }^{\mathrm{a}, \mathrm{c}}$, Emiko Tsugami ${ }^{\mathrm{b}}$, Shigenori Ando ${ }^{\mathrm{b}}$, Ayano Imai $^{\mathrm{b}}$, Suzuko Matsumoto ${ }^{\mathrm{a}}$, Takashi Omoto ${ }^{\mathrm{a}}$, \\ Masahiro Shinozaki ${ }^{a}$, Shinya Nishio ${ }^{\text {a }}$, Mariko Abe ${ }^{a}$, Shinichi Antoku ${ }^{a}$, Mizuo Mifune ${ }^{a}$, \\ Michiko Togane
}

\begin{abstract}
Background: We investigated the trends in the clinical characteristics and prescriptions of type 2 diabetic patients with severe hypoglycemia because the prescription rate of antidiabetic agents has significantly changed recently.
\end{abstract}

Methods: A total of 193 patients with type 2 diabetes with severe hypoglycemia induced by antidiabetic agents between 2008 and 2013 were divided into three groups based on the period of visit: 2008 2009, 2010 - 2011 and 2012 - 2013.

Results: While the proportion of patients with severe hypoglycemia using insulin (from $55 \%$ to $74 \%$ ), biguanides (from $6 \%$ to $20 \%$ ), glinides, and dipeptidyl peptidase- 4 inhibitors significantly increased, those using sulfonylureas (from $45 \%$ to $20 \%$ ) significantly decreased. Errors of drug use significantly increased as a trigger of hypoglycemia in recent years. The number of antidiabetic agents (from $1.9 \pm 0.6$ to $2.3 \pm 0.7$ ), non-diabetic agents (from $2.3 \pm 2.4$ to $4.3 \pm 3.3$ ), and total drugs prescribed were significantly higher in recent years among patients receiving insulin therapy.

Conclusions: Polypharmacy especially in patients receiving insulin therapy and errors of drug use have increased in type 2 diabetic patients with severe hypoglycemia in recent years. Intensive education in the usage rule of drugs is considered to be important in order to prevent severe hypoglycemia.

Keywords: Hypoglycemia; Insulin; Patient education; Polypharmacy; Sulfonylureas

\footnotetext{
Manuscript accepted for publication August 05, 2016

aDepartment of Diabetes, Metabolism and Kidney Disease, Edogawa Hospital, Tokyo, Japan

bepartment of Pharmacy, Edogawa Hospital, Tokyo, Japan

${ }^{\mathrm{c} C}$ Corresponding Author: Hiroyuki Ito, Department of Diabetes, Metabolism and Kidney Disease, Edogawa Hospital, 2-24-18, Higashikoiwa, Edogawa-ku, Tokyo 133-0052, Japan. Email: ito@edogawa.or.jp
}

doi: http://dx.doi.org/10.14740/jocmr2610w

\section{Introduction}

Hypoglycemia induced by antidiabetic agents is a serious problem for the treatment of patients with type 2 diabetes. In addition to subjects showing consciousness disturbance secondary to damage of the central nervous system, fatal cases have been reported in patients with severe hypoglycemia [1-3]. Furthermore, it has been described that a lack of awareness regarding hypoglycemia is frequently observed in patients with type 2 diabetes certain antidiabetic agents [3-5] and that hypoglycemia is associated with cognitive dysfunction [6]. Because recent clinical trials have demonstrated that intensive glycemic control worsens the prognosis of patients with type 2 diabetes due to the occurrence of cardiovascular events, which is considered to be associated with hypoglycemia, the achievement of a target blood glucose level to avoid hypoglycemia is currently recommended for the management of diabetes [7-12].

Due to the availability of a wide variety of antidiabetic agents, the prescription rate of antidiabetic agents has significantly changed in recent years [13-16]. Therefore, the details of prescription may also be diverse in diabetic patients with severe hypoglycemia, although hypoglycemia associated with antidiabetic agents is generally induced by an excessive effect of insulin and/or insulin secretagogues such as sulfonylureas (SU) and glinides. Thus, we hereby investigated the secular trends in the clinical characteristics and prescriptions of type 2 diabetic patients with severe hypoglycemia between 2008 and 2013.

\section{Materials and Methods}

Two hundred and six patients who were diagnosed with severe hypoglycemia were screened from 27,486 subjects who visited the emergency room of Edogawa Hospital, Tokyo, Japan, by ambulance between 2008 and 2013. After excluding subjects with type 1 diabetes $(n=7)$, without diabetes $(n=4)$ and whose medication was unknown $(\mathrm{n}=2), 193$ patients with type 2 diabetes receiving antidiabetic agents were investigated in the current study. All cases matched the definition of severe hypoglycemia proposed by the American Diabetes Association and the Endocrine Society [4], because they required the 
Table 1. Trends in the Clinical Characteristics and Prescriptions of Type 2 Diabetic Patients With Severe Hypoglycemia

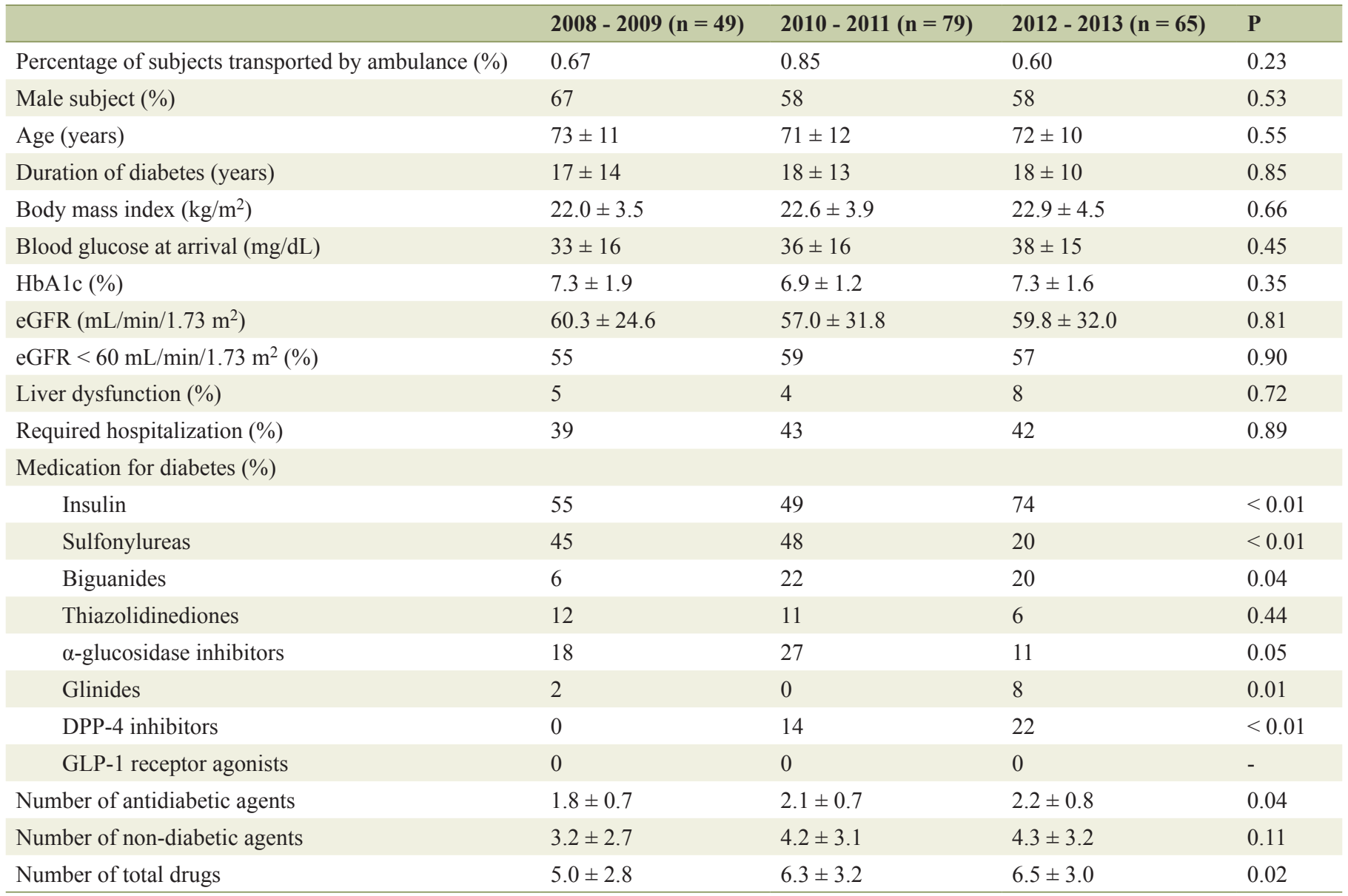

DPP-4: dipeptidyl peptidase-4; GLP-1: glucagon-like peptide-1. The P value was determined among the groups divided by the period of visit.

assistance of another person, such as emergency services, to actively administer carbohydrates, glucagon or take other corrective actions.

The study subjects were divided into three groups based on the period of visit: 2008 - 2009, 2010 - 2011 and 2012 2013. The clinical characteristics and prescriptions of the patients were investigated using the patients' medical records. Liver dysfunction was defined as both aspartate aminotransferase and alanine aminotransferase values $>40$ IU/L according to the reference values in our hospital. The necessity for hospitalization was judged by the physicians who examined the patients in the emergency room.

This study was conducted according to the principles expressed in the Declaration of Helsinki. The Ethics Committees of Edogawa Hospital approved the protocol of this study and waived the need for written informed consent because this cross-sectional study anonymously analyzed data stored in the hospital database (approval number: 2015-16).

\section{Statistical methods}

All data are presented as the mean $\pm \mathrm{SD}$. The Wilcoxon rank sum test and the $\chi^{2}$ test were used for between-group comparisons of continuous and categorical variables, respectively. Significant differences in the comparison of continuous variables among the three groups were assessed using the Kruskal-Wallis test. Differences of $\mathrm{P}<0.05$ (two-tailed) were considered to be statistically significant. The statistical software package JMP, version 8.0 (SAS Institute, Cary, NC, USA), was used to perform all of the analyses.

\section{Results}

Table 1 shows the trends in the clinical characteristics and prescriptions of the study subjects. The percentage of subjects transported by ambulance, male subjects, patients' age, duration of diabetes, body mass index (BMI), levels of blood glucose at arrival, $\mathrm{HbA1c}$, estimated glomerular filtration rate (eGFR) and prevalence of liver dysfunction did not differ among the groups according to the period of visit. While the proportion of subjects using insulin, biguanides, glinides and dipeptidyl peptidase (DPP)-4 inhibitors significantly increased in recent years, those receiving SU significantly decreased. The number of antidiabetic agents and total drugs, 
Table 2. Trends in the Triggers of Severe Hypoglycemia

\begin{tabular}{lllll}
\hline & $\mathbf{2 0 0 8 - 2 0 0 9}(\mathbf{n}=\mathbf{4 9})$ & $\mathbf{2 0 1 0}-\mathbf{2 0 1 1}(\mathbf{n}=\mathbf{7 9})$ & $\mathbf{2 0 1 2}-\mathbf{2 0 1 3}(\mathbf{n}=\mathbf{6 5})$ & $\mathbf{P}$ \\
\hline Decreased food intake (\%) & 39 & 37 & 34 & 0.86 \\
Errors of drug use (\%) & 18 & 28 & 37 & 0.04 \\
Alcohol consumption (\%) & 8 & 1 & 5 & 0.15 \\
Unknown (\%) & 33 & 29 & 22 & 0.34 \\
Others $(\%)$ & 2 & 5 & 3 & 0.63 \\
\hline
\end{tabular}

The $\mathrm{P}$ value was determined among the groups divided by the period of visit.

including non-antidiabetic agents, gradually increased significantly.

The trends in the triggers of severe hypoglycemia in the study subjects are shown in Table 2. Although a decreased food intake including diabetes sick days and alcohol consumption did not change, errors of drug use significantly increased in recent years.

When considering drugs mainly associated with hypoglycemia, insulin, SU, combination of insulin and SU and other antidiabetic agents were prescribed in 113,72 , one and seven patients, respectively. Table 3 shows the trends in the clinical characteristics and prescriptions of patients whose hypoglycemia was caused by insulin $(\mathrm{n}=113)$ and $\mathrm{SU}(\mathrm{n}=$ 72). The rate of required hospitalization gradually elevated among the subjects using SU. The total number of patients prescribed antidiabetic agents, non-diabetic agents and the total number of drugs significantly increased in recent years among patients receiving insulin therapy. Figure 1 demonstrates the scatter plots of the eGFR, patients' age, BMI and HbAlc value in patients with severe hypoglycemia catego- rized by antidiabetic agents (insulin or SU) over the entire study period. Subjects receiving SU tended to be elderly, have a low eGFR value, high BMI and low HbAlc level, while patients receiving insulin therapy did not show consistent trends.

\section{Discussion}

In the present study, the number of patients with severe hypoglycemia using insulin, biguanides, glinides and DPP-4 inhibitors significantly increased in recent years, while those receiving SU significantly decreased. Both the number of antidiabetic agents and total drugs gradually increased. Ambulatory treatment of type 2 diabetes has dramatically changed recently. Combination therapy of antidiabetic agents has continuously increased and the prescription pattern is now complicated [13-16], which are considered to be some reasons for the increasing number of agents prescribed for type 2 dia-

Table 3. Trends in the Clinical Characteristics and Prescriptions of Type 2 Diabetic Patients With Severe Hypoglycemia Caused by Insulin and Sulfonylureas

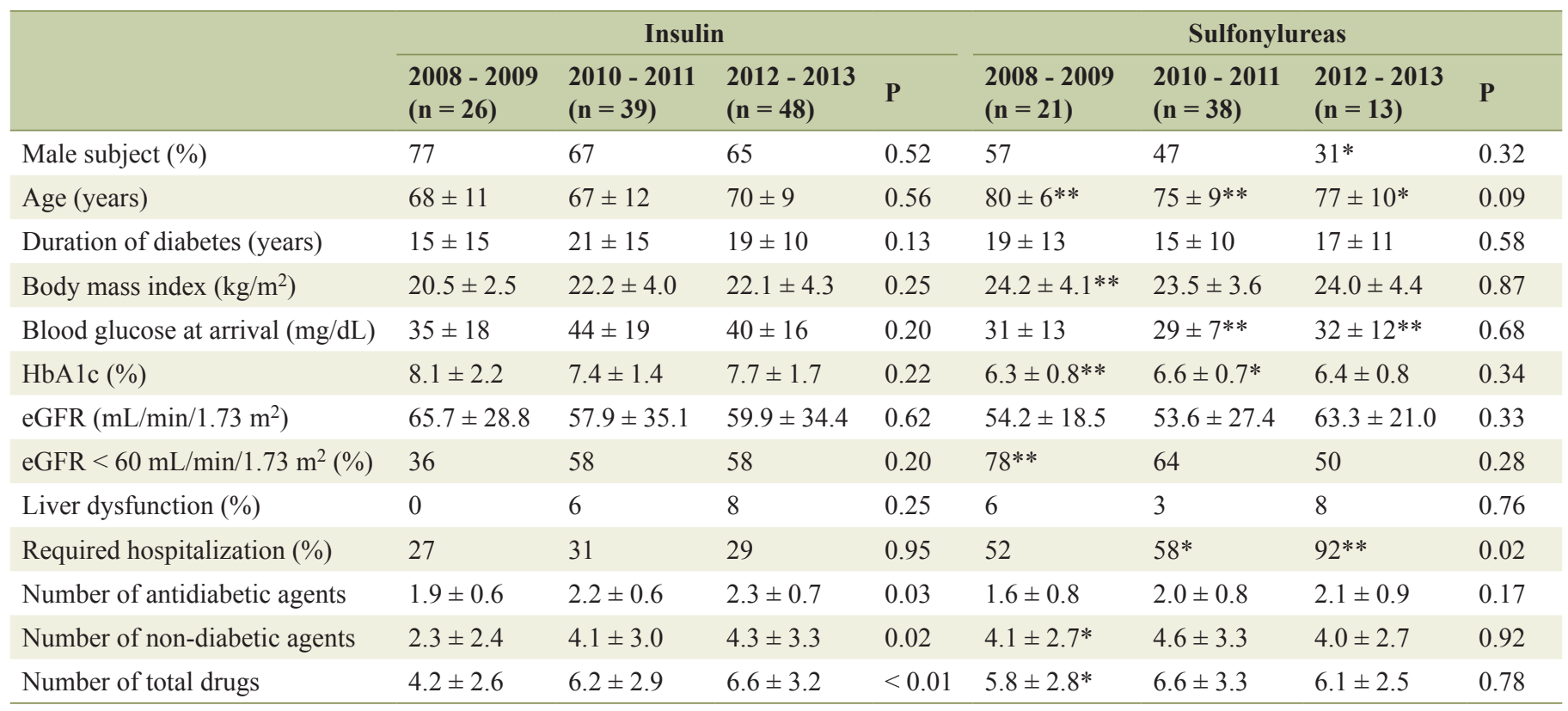

The $\mathrm{P}$ value was determined among the groups divided by the period of visit. ${ }^{*} \mathrm{P}<0.05$ and ${ }^{* *} \mathrm{P}<0.01$ vs. the corresponding value in patients receiving insulin therapy. 
(A)

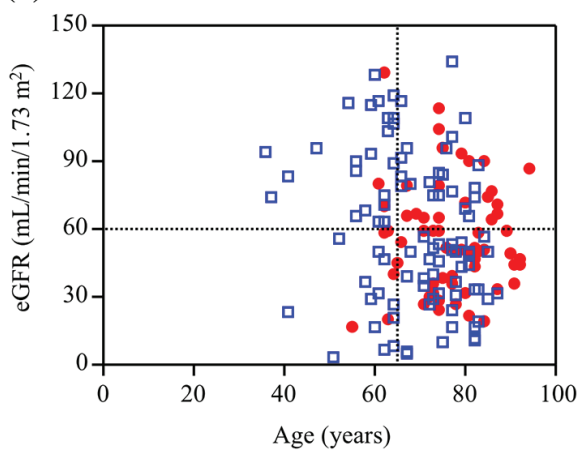

(B)

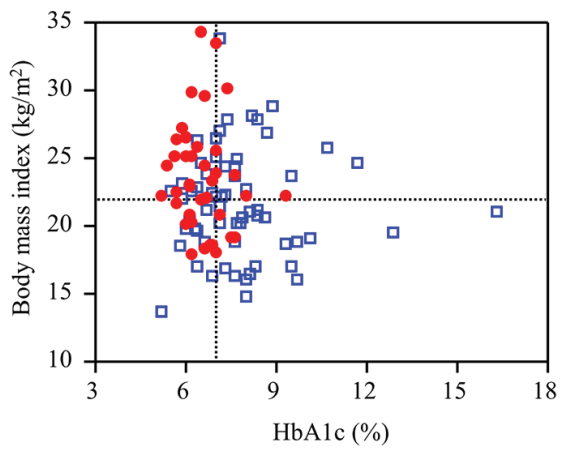

Figure 1. Scatter plots of (A) eGFR and patients' age and (B) body mass index and $\mathrm{HbA} 1 \mathrm{c}$ value categorized by the antidiabetic agents during the entire study period. Open blue squares and closed red circles indicate the patients with severe hypoglycemia induced by insulin and sulfonylureas, respectively.

betic patients with severe hypoglycemia. While the prescription rates of $\mathrm{SU}$ and $\alpha$-glucosidase inhibitors have decreased, those for DPP-4 inhibitors and biguanides have continually increased among outpatients with type 2 diabetes [13-16]. Similar trends were observed among hypoglycemic patients in the present study. Furthermore, the number of hypoglycemic patients receiving insulin therapy significantly increased in 2012 - 2013. Because the percentage of the subjects receiving insulin therapy did not change dramatically in recent years $[13,14]$, the increased number of patients with severe hypoglycemia associated with insulin therapy might be due to popularization of basal-bolus insulin therapy secondary to the development of insulin preparations and devices for injection.

Although there are many triggers for hypoglycemia in clinical practice, only errors of drug use have increased significantly in recent years according to the findings of the present study. This issue may be associated with the complexity of the prescription caused by the increase in the number of prescription drugs described above.

Various factors have been described to be risk factors for hypoglycemia associated with antidiabetic agents in patients with type 2 diabetes such as male sex, older age, long duration of diabetes, lower BMI, lower $\mathrm{HbAlc}$ level, renal dysfunction, previous hypoglycemia unawareness, cognitive dysfunction, peripheral neuropathy and concomitant medication [17-21]. In our present study, subjects receiving SU demonstrated characteristic features including older age, low eGFR value, high BMI and low HbAlc value, while those receiving insulin did not show consistent trends. Furthermore, the prevalence of patients who required hospitalization was significantly more frequent among subjects receiving SU compared with those receiving insulin therapy because it is well-known among physicians that hypoglycemia caused by $\mathrm{SU}$ is often prolonged especially in patients with renal impairment, compared to insulin-induced hypoglycemia $[22,23]$.

The number of antidiabetic agents, non-antidiabetic agents and total drugs was elevated in recent years among patients on insulin therapy. This is considered to reflect the notion that drug prescription is complicated in patients receiving insulin therapy. Therefore, intensive education in the usage rule of the drugs, including diabetes sick days, is especially important for type 2 diabetic patients treated with insulin in order to prevent severe hypoglycemia.

There are several limitations associated with the present study that should be kept in mind when interpreting the results. First, our study was a retrospective study with a rather small number of subjects at a single institution. Therefore, it is necessary to note the possibility that the statistical difference in the clinical parameters among the periods may have occurred incidentally due to the low statistical power. Second, our study did not include any subjects using sodium-glucose co-transporter 2 inhibitors, the latest oral antidiabetic agent which became available in April 2014 in Japan. Another investigation is necessary to determine the most recent condition of severe hypoglycemia. However, we believe that complicated prescriptions, including both antidiabetic and non-antidiabetic agents, and errors of drug use are now major problems for type 2 diabetic patients with severe hypoglycemia especially in subjects using insulin.

\section{Conclusions}

Polypharmacy and errors of drug use have increased in type 2 diabetic patients with severe hypoglycemia in recent years. Intensive education in the usage rule of the drugs, including diabetes sick days, is important in order to prevent the occurrence of severe hypoglycemia.

\section{Acknowledgments}

The authors thank Ms. Tomoko Koyanagi in the secretarial section and Ms. Kimiko Kobayashi in the Department of Nursing of Edogawa Hospital for their valuable help with data collection.

\section{Conflicts of Interest}

The authors declare that there are no conflicts of interest. 


\section{Grant Support}

None.

\section{Financial Disclosure}

None.

\section{References}

1. Asplund K, Wiholm BE, Lithner F. Glibenclamide-associated hypoglycaemia: a report on 57 cases. Diabetologia. 1983;24(6):412-417.

2. von Mach MA, Gauer M, Meyer S, Omogbehin B, Schinzel H, Kann PH, Weilemann LS. Antidiabetic medications in overdose: a comparison of the inquiries made to a regional poisons unit regarding original sulfonylureas, biguanides and insulin. Int J Clin Pharmacol Ther. 2006;44(2):51-56.

3. Cryer PE, Davis SN, Shamoon H. Hypoglycemia in diabetes. Diabetes Care. 2003;26(6):1902-1912.

4. Seaquist ER, Anderson J, Childs B, Cryer P, DagogoJack S, Fish L, Heller SR, et al. Hypoglycemia and diabetes: a report of a workgroup of the American Diabetes Association and the Endocrine Society. Diabetes Care. 2013;36(5):1384-1395.

5. Ito $\mathrm{H}$, Abe M, Shinozaki M, Oshikiri $\mathrm{K}$, Yamashita $\mathrm{H}$, Nakashima M, Takaki A, et al. Hypoglycemia observed during continuous glucose monitoring in patients with type 2 diabetes mellitus treated by subcutaneous insulin injection. Diabetes Technol Ther. 2013;15(7):586-590.

6. Whitmer RA, Karter AJ, Yaffe K, Quesenberry CP, Jr., Selby JV. Hypoglycemic episodes and risk of dementia in older patients with type 2 diabetes mellitus. JAMA. 2009;301(15):1565-1572.

7. Duckworth W, Abraira C, Moritz T, Reda D, Emanuele N, Reaven PD, Zieve FJ, et al. Glucose control and vascular complications in veterans with type 2 diabetes. $\mathrm{N}$ Engl J Med. 2009;360(2):129-139.

8. Gerstein HC, Miller ME, Byington RP, Goff DC, Jr., Bigger JT, Buse JB, Cushman WC, et al. Effects of intensive glucose lowering in type 2 diabetes. N Engl J Med. 2008;358(24):2545-2559.

9. Patel A, MacMahon S, Chalmers J, Neal B, Billot L, Woodward M, Marre M, et al. Intensive blood glucose control and vascular outcomes in patients with type 2 diabetes. N Engl J Med. 2008;358(24):2560-2572.

10. Hemmingsen B, Lund SS, Gluud C, Vaag A, Almdal T, Hemmingsen C, Wetterslev J. Intensive glycaemic control for patients with type 2 diabetes: systematic review with meta-analysis and trial sequential analysis of randomised clinical trials. BMJ. 2011;343:d6898.

11. Boussageon R, Bejan-Angoulvant T, Saadatian-Elahi M, Lafont S, Bergeonneau C, Kassai B, Erpeldinger S, et al. Effect of intensive glucose lowering treatment on all cause mortality, cardiovascular death, and microvascular events in type 2 diabetes: meta-analysis of randomised controlled trials. BMJ. 2011;343:d4169.

12. Goto A, Arah OA, Goto M, Terauchi Y, Noda M. Severe hypoglycaemia and cardiovascular disease: systematic review and meta-analysis with bias analysis. BMJ. 2013;347:f4533.

13. Nishio S, Abe M, Ito H. Anagliptin in the treatment of type 2 diabetes: safety, efficacy, and patient acceptability. Diabetes Metab Syndr Obes. 2015;8:163-171.

14. Turner LW, Nartey D, Stafford RS, Singh S, Alexander GC. Ambulatory treatment of type 2 diabetes in the U.S., 1997-2012. Diabetes Care. 2014;37(4):985-992.

15. Oishi M, Yamazaki K, Okuguchi F, Sugimoto H, Kanatsuka A, Kashiwagi A. Changes in oral antidiabetic prescriptions and improved glycemic control during the years 2002-2011 in Japan (JDDM32). J Diabetes Investig. 2014;5(5):581-587.

16. Kohro T, Yamazaki T, Sato H, Harada K, Ohe K, Komuro I, Nagai R. Trends in antidiabetic prescription patterns in Japan from 2005 to 2011. Int Heart J. 2013;54(2):93-97.

17. Haneda M, Morikawa A. Which hypoglycaemic agents to use in type 2 diabetic subjects with CKD and how? Nephrol Dial Transplant. 2009;24(2):338-341.

18. Lipska KJ, Warton EM, Huang ES, Moffet HH, Inzucchi $\mathrm{SE}$, Krumholz HM, Karter AJ. HbAlc and risk of severe hypoglycemia in type 2 diabetes: the Diabetes and Aging Study. Diabetes Care. 2013;36(11):3535-3542.

19. Yanai H, Adachi H, Katsuyama H, Moriyama S, Hamasaki H, Sako A. Causative anti-diabetic drugs and the underlying clinical factors for hypoglycemia in patients with diabetes. World J Diabetes. 2015;6(1):30-36.

20. Ahren B. Avoiding hypoglycemia: a key to success for glucose-lowering therapy in type 2 diabetes. Vasc Health Risk Manag. 2013;9:155-163.

21. Sako A, Yasunaga H, Matsui H, Fushimi K, Hamasaki H, Katsuyama H, Tsujimoto T, et al. Hospitalization for Hypoglycemia in Japanese Diabetic Patients: A Retrospective Study Using a National Inpatient Database, 20082012. Medicine (Baltimore). 2015;94(25):e1029.

22. Yale JF. Oral antihyperglycemic agents and renal disease: new agents, new concepts. J Am Soc Nephrol. 2005;16(Suppl 1):S7-10.

23. Abe M, Okada K, Soma M. Antidiabetic agents in patients with chronic kidney disease and end-stage renal disease on dialysis: metabolism and clinical practice. Curr Drug Metab. 2011;12(1):57-69. 